\title{
A review on Applications of Principles of Medical Ethics in the Practice of Surgery
}

\author{
Shaha, K.K. ${ }^{1}$, Patra, A.P. ${ }^{1}$, Chaudhari, V.A. ${ }^{1}$, Das, $S .{ }^{1}$, Das, S.K. ${ }^{2}$
}

\begin{abstract}
This review article is based on concepts of medical ethics related to the day-to-day practice of surgery. Over the last two decades, with the development of advanced surgical methods, robotic surgeries, or telesurgeries, etc. there is increased reporting of associated ethical issues as well. Nevertheless, the ethical issues encountered by surgeons are reasonably different and critical when compared to what physicians face during their practice. There are extensive deontology literatures available for physicians while the same is not available sufficiently for surgeons. In this review, we have discussed the common queries asked by practicing surgeons who had faced medico-legal litigation and had come to us for consultation. This discussion is made in the light of comparison with ethical issued faced by practicing physicians. In the practice of medicine just fulfilling the basic ethical requirements, e.g. autonomy, beneficence, non-maleficence, is often sufficient. However, for surgical practice the ethical requirement is beyond the level of such basic deontological requirements. Hence, in this paper we have tried to search and discuss all deontological dimensions of surgical practice, e.g. Normative ethics: concerned with a moral course of action for a surgical practice, Meta-ethics: concerning determining the truth and reference of moral values, and the Applied ethics: concerning what a person is obligated to do in a specific situation or a particular domain of action. Hopefully, these will be useful to guide the surgeons' behaviours in particular circumstances encountered during their surgical practice.
\end{abstract}

Keywords: normative ethics; meta-ethics; applied ethics; autonomy; beneficence; non-maleficence; justice; surgery

\section{Introduction}

In common surgical practice, a surgeon has the ethical responsibility to look after not only the disease proper, but also the patient's overall wellbeing and often that of the anaesthetist as well. Usually, subsequent to some surgical complications the litigations usually brought against the surgeons first instead of the anaesthetist.

There is common consensus that surgeons are responsible for all activities related to patient's treatment and care in surgical units

\footnotetext{
${ }^{1}$ Department of Forensic Medicine, JIPMER, Pondicherry, India

${ }^{2}$ Department of Forensic Medicine, AlIMS, Raipur, India

Corresponding Author:

Kusa Kumar Shaha,

Additional Professor\& HOD, Department of Forensic

Medicine, JIPMER, Pondicherry, India
}

Email:drkusashaha@yahoo.com
Therefore, it is important for them to act in the best and correct way towards patients, relatives, and their colleagues (Uden et al., 1992; LaSalle, 1997; Lindseth, 1992).

"What I have done wrong for which I am facing this litigation?"

"I was too busy. Despite that, I think I have acted reasonably, isn't it?"

\section{"What could I have done differently to avoid it?"}

These are some frequently encountered lines from surgeons facing litigations and seek assistance from the Department of Forensic Medicine for medico-legal consultation. These lines reflect the ethical dilemma and the deontological concept showed by an average surgeon. These questions identify the surgeon as the agent who potentially could have

DOI: http://doi.org/10.4038/seajme.v12i1.37

(CC) $\begin{aligned} & \text { C SEAJME. This is an Open Access article distributed under the terms of the Creative Commons Attribution } \\ & \text { License (http://creativecommons.org/licenses/by/4.0/), which permits unrestricted use, distribution, and } \\ & \text { reproduction in any medium, provided the original author and source are credited }\end{aligned}$


avoided the complications. Social Scientist Charles Bosk, three decades ago ascertained that surgical treatment links the behaviour of the surgeon and the response of the patient more closely than other aspects of medicine (Kodner et al., 2007).

The ethical issues and responsibilities associated with surgeons are reasonably different and more serious compared to those associated with practicing physicians. We are citing the following questions we encountered during our medico-legal practice in two different scenarios:

1. "How did the patient die?", the regular question asked by the associates once a patient dies in Medicine.

2. "See, what did he do?", the same situation in case of surgeon where his patient dies.

This shift reflects the distinction in the way surgeons conceptualize their responsibilities to their patients. However, it is not mandatory that a surgery will be free of complications. For example, most surgeons have heard the saying, "the only surgeon without complications is the surgeon who does not operate." Nevertheless, complications put an essential weight on surgeons by virtue of its own merit. Surgical complication is an inevitable part of surgical practice irrespective the place or period. In spite of this importance and prevalence of the issue, there is at present no clear, consistent or acceptable definition of a surgical complication (Bosk, 1979; Rampersaud, 2006). However in the World Journal of Surgery: "A surgical problem is any undesirable, unintended, and direct outcome of surgery which affect the patient, which would not have occurred had the surgery gone as well as could reasonably be hoped" (Sokol \& Wilson, 2008). The objective of this article is to outline the key ethical issues from the surgeon's point of view relating to surgical complications as opposed to that of other members of the surgical team.

\section{Background}

In 1970s, American philosophers Tom Beauchamp and James Childress introduced the highly influential "Four Principles" approach to medical ethics (Beauchamp \& Childress, 2001). To guide the surgeons' behaviors and define the surgeon's proper role, the four widely known ethical principles the value for the autonomy, beneficence, nonmaleficence, and justice can be applied in particular circumstances. Although the broad principles appear to be accepted from all over cultures, the priority of these principles may vary among different cultures. The principles of ethics are neither hierarchically ordered nor invariably binding.

Ethical considerations cannot be avoided by surgeons in courses of action that are available for patients in particular situations (Nandi, 2000; Mclntyre \& Popper, 1983). A short depiction of the Ethical Principles are as follows (Beauchamp \& Childress, 2001).

1. Respect for Autonomy: autonomy in this context means the patient's right and ability to make an informed choice based on their beliefs and values about, whether to have an accurate procedure. Doctors should give adequate information to support patients in their decision-making.

2. Beneficence: surgeon's obligation is to provide benefit effective treatments by acting in patients best interests. The conception of what constitutes benefit and harm differs from individual to individual

3. Non-maleficence: all efforts to benefit patients, whether through words, medications or techniques, carry risks of harm. Non-maleficence denotes moral obligation to avoid causing not harm to patients by providing safe treatments and should be thought in combination with the principle of beneficence.

4. Justice: to distribute scarce health care resources fairly for patients in similar circumstances.

These 'four' broad principles create "obtain informed consent," "respect confidentiality," good communication skills, ability to exercise sound judgment and disclosure which act as a system to consider the ethics of surgical complications systematically.

\section{Discussion}

Uses of the 'four principles approach' in surgery

\section{Respect for autonomy}

The surgeon's role is said to have changed from paternalism with regard to patients' autonomy (Guadagnoli \& Ward, 1998). Patients are able to think about a decision, receive the exact information about their condition, the nature of the proposed 
treatment, prognosis, the options, risks and benefits the way he or she would understand. It is the way surgeons acknowledge patients' rights. Most often the final decisions are made by surgeons even when patients were well informed (Charles \& Gafni, 1999; Falkum \& Forde, 2000). In an emergency, patient choices may be uncertain, with less time to determine them. In this particular situation, it is prudent to give standard medical care (Guadagnoli \& Ward, 1998).

\section{i. How much information is adequate?}

ii. More than the required information to the patient may spoil the actual goal of the principle of autonomy by confusing or putting the patient into a dilemma of choices.

iii. Excessive information about remote possible or theoretical complications may frighten the patient and he may refuse a desirable surgery.

It is hard to evaluate patients' understanding of the surgeons' information when patients volunteer their consent or delegate the decision-making to the surgeon without having understood the consequences due to confidence and faith in surgeon (British Medical Association, 2002). Although the surgeon spends a lot of time rendering information, it may not be certain that the patients fully understand it. It is challenging to perform operations when they were in uncertainty about patients' capacity to make an informed choice. Clear disclosure of the patient's situation requires a combination of good judgment and communicative ability. Components affecting the extent of disclosure:

i. The extent of the complication (more serious the complication, the more inclined the surgeon should be to divulge it)

ii. The chance of the complication (disclose to the patient, prior to surgery prevent more likely complication)

iii. The patient's information preferences needs and circumstances of the individual patient.

Some courts have applied the "reasonable person standard" to decide how much information should be given (Department of Health, 2001). This standard requires the surgeon to present information that a reasonable person in the patient's circumstances would want to know. The surgeon must be ready to justify his or her decision to the courts when patient requires an unplanned procedure intra-operatively, and explicit consent has not been acquired. It is advisable to take more general consent or to appoint a proxy to act on the patient's behalf, where patients cannot be informed to treat any unforeseen circumstance emerges during the surgery. It is always wise to consult and take the advice of an experienced colleague whenever required.

There is a decrease in surgeon's autonomy because more external factors influence decisions concerning patients' treatment (Nandi, 2000; McCullough, 1998). Surrogates, appearing on behalf of patients who do not have decision-making power, may not always accurately reflect the patients' preferences (Nichol et al., 1999). The doctor in advance discusses the subject with the patient at the time of admission to hospital and help to establish patient desire, but patients must not be enforced into providing advance directives. The surgeon should provide a balanced presentation of information to ensure that patients are actually informed and educated before consenting (Lieberman \& Wenger, 2004; Gates, 1997; ACOG, 2006).

\section{Disclosing complications (truth-telling and openness)}

For entitlement to compensation, within the principle of justice, the patient has a privilege to know the information in regards to mistakes made by a surgeon who tries to conceal it by calling it an error or complication (Faraj \& Drakau, 2007). Patients who demand to have their operation performed by a consultant, rather than a junior doctor, have their requests permitted on the basis of respecting autonomy and in the principle of beneficence. Withholding vital information to the patient with respect to the operation by trainee where the patient believes that the senior surgeon will do is a form of deception by omission.

\section{Beneficence}

A surgeons' responsibility is to save the life. Does ethics centre on difficult ethical dilemmas and decision making to justify our behaviours and give answers to the questions like: What should I (we) do? Did I (we) make the best decision in this situation? (Lindseth, 1992). The surgeon's desire to introduce new surgical procedures because they tend to be 
less invasive, shorter operative times, less costly than established procedures and benefit with respect to patients (Gates, 1997; Norton, 2006). For the patients benefit and to reduce the likelihood of complications, surgeons must be knowledgeable in surgical techniques and competent in the ability to recognize the limits of their professional competence. Surgeons should refer the patient to specialist colleagues in cases deemed beyond their expertise (Peterson, 2000). In order to gain the trust and confidence of the patient the surgeon provides all available facts which eases to obtain the informed consent (Ostergard, 2007). If surgeons have doubts about the effectiveness and safety of the treatment, they should not use the new method (Iserson \& Chiasson, 2002). The appropriateness of surgery needs good surgical judgment to assess the risks, burdens, and benefits. Ethical principles mandate that surgeons should honestly provide full information on the effectiveness, safety, and rates of complications of the proposed treatment (Canadian Medical Protective Association, 1996).

Surgeons have a duty to stop operating where they have personal health problems or substandard health. The likelihood of surgical complications because of human error is likely to increase with minimal sleep, poor concentration, stress, and other physical and psychic illness. The surgeon should ensure that appropriate infection control measures have been taken to reduce the incidence of iatrogenic complications. Surgeons should ensure that the equipment is functioning and reliable. Faulty equipment compromises patient care and increases the likelihood of surgical complications.

Patients will benefit, and the rate of complications will reduce if surgeons update their knowledge by attending conferences, going through journals, and always keep with the recent therapeutic developments in their field. Regrettably, attending courses and meetings is increasingly difficult at a time of shortened study leave and reduced budgets. Surgeon's professional and educational advancement is necessary for ensuring the highest standards of patient care and the lowest rates of complications, what Sir William Osler called the "quality of thoroughness" (Osler, 1928).

Due to the advancement of scientific knowledge, developed medical technology, patients-surgeons relationship, changes in the organizational setup and financing of the health care system, today surgeons are faced with more ethical dilemmas than before (Nandi, 2000; McCullough et al., 1998; Hanlon, 1998; Torjuul, 2005).

\section{Non-maleficence}

The majority of the ethical issues discussed under the principle of beneficence-are recognition of the limits of one's expertise, the potential to exercise good judgment, strict contamination control measures, and the need for research. In toto all add to reduce the event of surgical complications and subsequently likewise fall under the principle of nonmaleficence. Surgeons should communicate legitimately to patients as well as to other members of the team. Sometimes poor communication about risks, benefits and complications before and after surgery leads to emotional distress inpatients. Substandard communication such as unclear or insufficiently detailed instructions can also lead to prompt error, misunderstandings within the surgical team (Beauchamp \& Childress, 2001). Risk-free surgery requires good teamwork and fear, stress, or anger may easily lead individuals to make mistakes. Surgeons have a moral obligation to minimize recurrence of similar complications by reviewing in regular "mortality and morbidity" meetings. The others surgeon may benefit from the experience of the surgeon who faces the surgical complications by publishing in journal articles or presenting at the conferences. Surgeons have a dual liability for their conduct in particular situations (indicating respect and empathy for patients) (Peterson, 2000; Nerheim, 1991).

\section{Justice}

Detailed examinations of a single patient would prevent the resources available for other patients if the clinical signs and manifestations did not relate to the patients' complaints. If a surgeon realizes a problem in colleagues that may put patients at unnecessary risks, such as alcoholism or drug dependence, he or she has an ethical obligation to inform higher authority (Moore, 2000; Veen et al., 1999). There is an economic burden to the patients for longer hospital stays. Finally, patients may be entitled to legitimate help and financial compensation in the event of negligent surgical complications. 


\section{Conclusion}

This outline of the ethics of surgical complications has been primarily concerning moral perception-discussing why \& how surgeons have duties toward patients making utilization of the four principles. The ethical responsibility arises from the ethical demands which are inherent to the patient-physician relationship.

The role of the clinician is said to require both the ability and courage to act in dubious and high-risk instances where the outcomes may be marginal. Notification of death and the discussions that take after are difficult, even for skillful health care providers. Survivors should be told with understanding by a skilled knowledgeable healthcare professional. Healthcare professionals can be sensitive to survivors' needs by using appropriate words and acceptable body language. No one but time can tell whether surgeons do rise to the challenge to improve ethical standards.

\section{Acknowledgement}

We acknowledge and dedicate our indebtedness to those medical practitioners who faced ethical dilemmas and approached us for consultation during our practice of Forensic Medicine \& Medical Jurisprudence.

\section{Competing interests}

All authors declare no competing interests.

\section{References}

American College of Obstetricians and Gynecologists Committee on Ethics, (2006) ACOG Committee Opinion No. 352: Innovative practice: ethical guidelines, Obstetricians \& Gynecologists, 108, 6, pp. 1589-1595.

Beauchamp, T. \& Childress, J. (2001) Principles of biomedical ethics, New York: Oxford University Press.

Bosk, C.L. (1979) Forgive and remember: managing medical failure, University of Chicago Press Chicago, pp. 30.

British Medical Association (2002) Medical ethics today, London: BMJ Publishing Group.

Canadian Medical Protective Association (1996) Consent: a guide for Canadian physicians [3rd Ed.] Ottawa: Canadian Medical Protective Association.
Charles, C., Gafni, A. \& Whelan, T. (1999) Decision-making in the physician patient encounter: revisiting the shared treatment decision making model, Social Science \& Medicine, 49, 5, pp. 651-661.

Britain, G. (2001) Good Practice in Consent Implementation Guide: Consent to Examination or Treatment, Department of Health, DoH Publications: London.

Falkum, E. \& Førde, R. (2001) Paternalism, patient autonomy, and moral deliberation in the physician - patient relationship, Social Science \& Medicine, 52, 2, pp. 239-248.

Faraj, A. \& Drakau, N. (2007) Press-fit hemiarthroplasty for elderly with femoral neck fracture: high complication rates in operations performed by younger surgeons, European Journal of Orthopaedic Surgery \& Traumatology, 17, 3, pp. 267-271.

Fletcher, L. \& Buka, P. (1999) A legal framework for caring: an introduction to law and ethics in health care, Basingstoke: Palgrave.

Gates, E.A. (1997) New surgical procedures: can our patients benefit while we learn? American Journal of Obstetrics Gynecology, 176, 6, pp. 1293-1298.

Guadagnoli, E. \& Ward, P. (1998) Patient participation in decision-making, Social Science \& Medicine, 47, 3, pp. 329-339.

Hanlon, C.R. (1998) Ethics in surgery, Journal of the American College of Surgeons, 186, 1, pp. 4149.

Iserson, K.V. \& Chiasson, P.M. (2002) The ethics of applying new medical technologies, In.Seminars in Laparoscopic Surgery, 9, 4, pp. 222-229.

Kodner, I.J., Siegler, M., Choctaw, W., Wolff, B.G., Fleshman, J.W., Beck, D.E., Pemberton, J.H., Wexner, S.D., (2007) (eds): The ASCRS Textbook of Colon and Rectal Surgery, New York: Springer-Verlag c, pp. 29.

LaSalle, D.L. (1997) Ethics in research and surgical practice, The American Journal of Surgery, 174, 6, pp. 589-591.

Lieberman, J.R. \& Wenger, N. (2004) New technology and the orthopaedic surgeon: are you protecting your patients? Clinical Orthopaedics \& Related Research, 429, pp. 338-341.

Lindseth, A. (1992) The role of caring in nursing ethics, In Quality development in nursing care. From practice to science Edited by: Udén, G. Linköping: University Health Service Studies, pp. 97-106. 
McCullough, L.B., Jones, J.W. \& Brody, B.A. [Eds] (1998) Surgical Ethics, New York, Oxford: Oxford University Press.

Mclntyre, N. \& Popper, K. (1983) The critical attitude in medicine: the need for a new ethics, BMJ: British Medical Journal, 287, 6409, pp. 1919-1923.

Moore, F.D. (2000) Ethical problems special to surgery: surgical teaching, surgical innovation, and the surgeon in managed care, Archives of Surgery, 135, 1, pp. 14-16.

Veen, M.R., Lardenoye, J.W.H., Kastelein, G.W. \& Breslau, P.J. (1999) Recording and classification of complications in a surgical practice, European Journal of Surgery, 165, 5, pp. 421-424.

Nandi, P.L. (2000) Ethical aspects of clinical practice, Archives of Surgery, 135, 1, pp. 2225.

Nerheim, H. (1991) Theethical foundational experience from understanding rules to experiential knowledge, Universitetsforlaget AS Oslo, Norwegian.

Nichol, G., Stiell, I.G., Hebert, P., Wells, G.A., Vandemheen, K. \& Laupacis, A. (1999) What is the quality of life for survivors of cardiac arrest? A prospective study, Academic Emergency Medicine, 6, 2, pp. 95-102.

Norton, P. (2006) New technology in gynaecologic surgery: is new necessarily better? Obstetrics \& Gynecology, 108, 3, pp. 707-708.
Osler, W. (1928) Teacher and student. In: Aequanimitas with other addresses to medical students, nurses and practitioners of medicine, Blakiston, Philadelphia, pp. 21-43.

Ostergard, D.R. (2007) Lessons from the past: directions for the future. Do new marketed surgical procedures and grafts produce ethical, personal liability, and legal concerns for physicians? International Urogynecology Journal, 18, 6, pp. 591-598.

Peterson, L.M. (2000) Human values in the care of the surgical patient, Archives of Surgery, 135, 1, pp. 46-50.

Rampersaud, Y.R., Moro, E.R., Neary, M.A., White, K., Lewis, S.J., Massicotte, E.M. \& Fehlings, M.G. (2006) Intraoperative adverse events and related postoperative complications in spine surgery: implications for enhancing patient safety founded on evidence-based protocols, Spine, 31, 13, pp. 1503-1510.

Sokol, D. \& Wilson, J. (2008) What is a surgical complication? World Journal of Surgery, 32, pp. 942-944.

Torjuul, K., Nordam, A. \& Sørlie, V. (2005) Ethical challenges in surgery as narrated by practicing surgeons, BMC Medical Ethics, 6, 1, pp. 2.

Uden, G. Norberg, A., Lindseth, A. \& Marhaug, V. (1992) Ethical reasoning in nurses' and physicians' stories about care episodes, Journal of Advanced Nursing, 17, 9, pp. 10281034. 